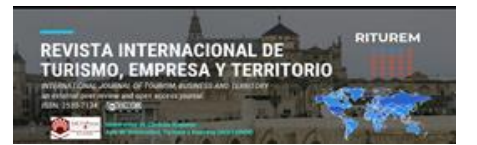

Cita bibliográfica: Sancho, A. y Serrat, R. (2020). Diseño y aplicación de un método para la creación de redes de itinerarios ecoturísticos. Una experencia en territorios de montaña mediterránea. Revista Internacional de Turismo, Empresa y Territorio, 4 (1), 22-41. https://doi.org/10.21071/riturem.v4i1.12710

\title{
Diseño y aplicación de un método para la creación de redes de itinerarios ecoturísticos. Una experiencia en territorios de montaña mediterránea
}

\section{Designing and implementing a method for the creation of networks of eco-touristic routes. Some lessons from Mediterranean mountain areas}

\author{
Alexis Sancho Reinoso ${ }^{1^{*}}$ \\ Ramon Serrat i Mulà ${ }^{2}$
}

\section{Resumen}

En el marco de un proyecto transnacional, la red de montañas emblemáticas mediterráneas (EMM, por sus siglas en inglés) está desarrollando el diseño y estructuración de nueve ecoviajes como producto turístico basado en rutas por territorios situados alrededor de áreas de montaña cercanas a la costa mediterránea. Los ecoviajes nacen con la intención de generar una alternativa al turismo de masas que soporta tanto el litoral como - en ocasiones - las cimas de las montañas. En el presente artículo, describimos el modelo y el proceso de diseño y desarrollo sobre el terreno de estos ecoviajes. Además, interpretamos algunos resultados de dicho proceso; que muestran la complejidad que supone el trabajo en red, además de poner de manifiesto los numerosos retos que plantea la práctica del turismo sostenible en territorios rurales y de montaña, tanto para actores públicos como privados.

Palabras clave: ecoturismo, cooperación, actores locales, desarrollo local, ecoviaje, montaña mediterránea.

\begin{abstract}
Within the framework of a transnational project, the Emblematic Mediterranean Mountains network (EMM) has developed nine ecojourneys as a tourist product based on routes through areas located in the hinterland of mountain regions near the Mediterranean coast. The ecojourneys intend to become an alternative to mass tourism that affects both the coastline and - sometimes- mountain summits. In this article, we describe the design process and development on the field of the ecojourneys. In addition, we interpret the results of the process. They show the complexity of networking, in addition to highlighting the numerous challenges that applying the principles of sustainability in tourism pose in rural and mountain territories, both for public and private actors.
\end{abstract}

Keywords: ecotourism, cooperation, local stakeholders, local development, ecojourney, Mediterranean mountain areas.

1 Departamento de Antropología Social y Cultural, Universidad de Viena, Austria. alexis.sancho.reinoso@univie.ac.at. Id orcid: 0000-0001-8535-0939.* Autor para la correspondencia

${ }^{2}$ Barcelona School of Tourism, Hospitality and Gastronomy, Barcelona, España. ramon.serrat@ cett.cat. Id orcid: 0000-0002-4691-2131. 


\section{Introducción}

\subsection{Presentación}

El presente artículo recoge el conjunto de experiencias que la Barcelona School of Tourism, Hospitality and Gastronomy, centro adscrito a la Universidad de Barcelona (de ahora en adelante CETT-UB), obtuvo de su colaboración con la Red de montañas emblemáticas del Mediterráneo (EMM, por sus siglas en inglés); una colaboración que se materializó en el marco del proyecto europeo EMbleMatiC (Emblematic Mediterranean Mountains as Coastal Destinations of Excellence).

En 2013 comenzó su andadura la Red EMM, cuando cuatro entidades procedentes de regiones montañosas de Francia, Grecia y Bulgaria emprendieron un proyecto conjunto financiado por el programa de desarrollo local LEADER. Dichas entidades identificaron tres retos comunes que decidieron afrontar de forma conjunta: (i) preservar los valores patrimoniales de amenazas internas y externas; (ii) compartir experiencias positivas y buenas prácticas; y (iii) incrementar el reconocimiento internacional. Además, reconocieron un conjunto de valores compartidos: se identificaron a sí mismos como territorios "emblemáticos" que cuentan con paisajes pintorescos, mitos y leyendas, fuerza de inspiración para artistas, lugares de relevancia histórica, altas cotas de biodiversidad y servicios locales específicos. En 2015, la red se amplió hasta los actuales nueve miembros (Fig. 1), que, entre 2017 y 2019, trabajaron en el marco del proyecto EMbleMatiC, financiado por el programa INTERREG. El consorcio estuvo formado por diez socios (Tabla 1): los nueve miembros de la red más el CETT-UB, que participó como socio académico.

Figura 1. Mapa de localización de los territorios de referencia del proyecto EMbleMatiC

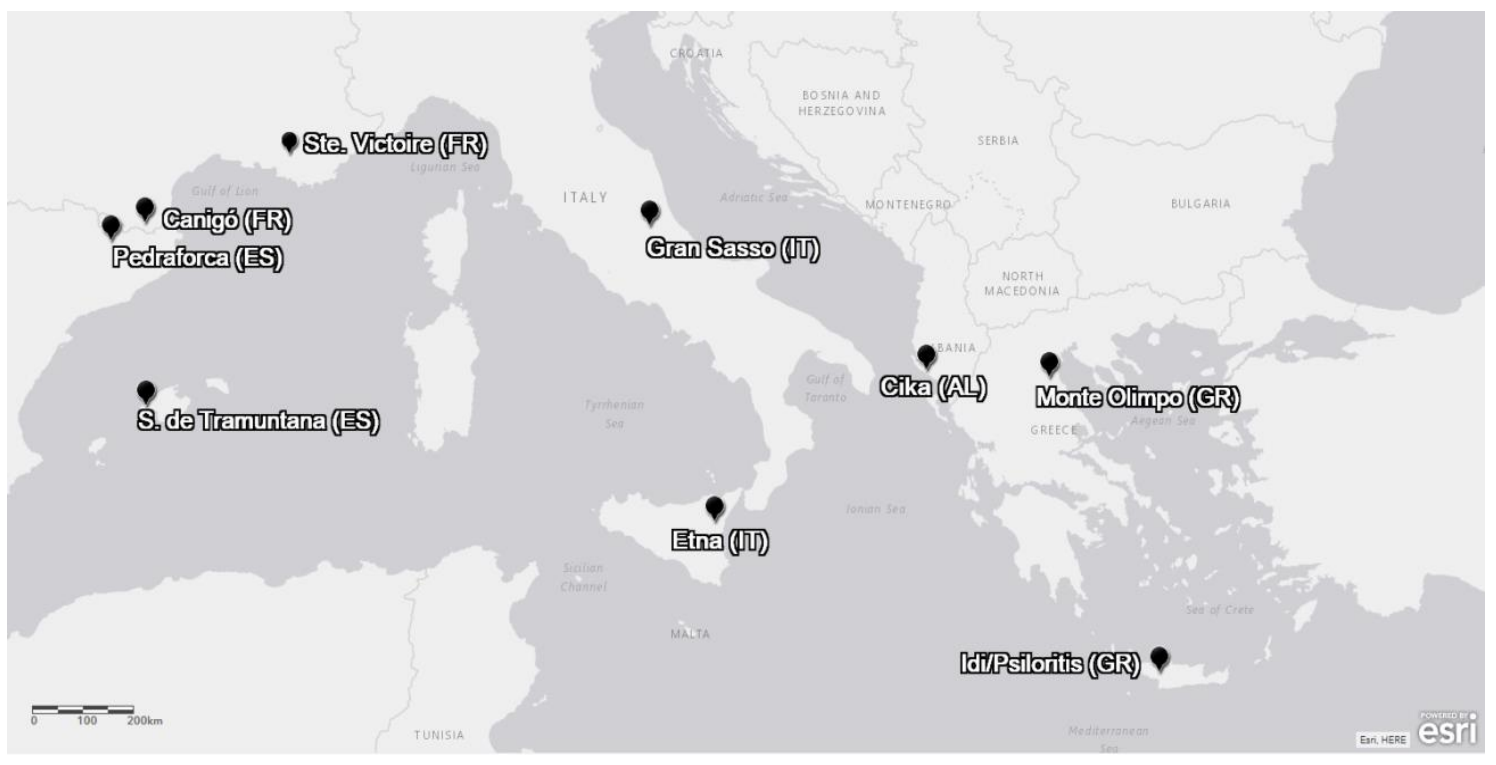

Fuente: Elaboración propia a partir de la documentación del proyecto. 
Tabla 1. Socios del proyecto EMbleMatiC.

\begin{tabular}{|l|l|l|}
\hline \multicolumn{1}{|c|}{ Nombre de la entidad } & \multicolumn{1}{|c|}{$\begin{array}{c}\text { Montaña de } \\
\text { referencia }\end{array}$} & \multicolumn{1}{|c|}{ Región (estado) } \\
\hline $\begin{array}{l}\text { Syndicat Mixte du Canigó } \\
\text { (coordinador) }\end{array}$ & Canigó & Occitania (FR) \\
\hline Municipio de Himara & Çika & (AL) \\
\hline $\begin{array}{l}\text { Agència de Desenvolupament del } \\
\text { Berguedà }\end{array}$ & Pedraforca & Cataluña (ES) \\
\hline GAL Gran Sasso Velino & Gran Sasso & Abruzos (IT) \\
\hline Pieriki Anaptixiaki & Olympus & $\begin{array}{l}\text { Macedonia Central } \\
\text { (GR) }\end{array}$ \\
\hline GAL Terre dell'Etna e dell'Alcantara & Etna & Sicilia (IT) \\
\hline Consell Insular de Mallorca & Serra de Tramuntana & Islas Baleares (ES) \\
\hline $\begin{array}{l}\text { AKOMM-Psiloritis Development } \\
\text { Agency }\end{array}$ & Idi (Psiloritis) & Creta (GR) \\
\hline Métropole d'Aix-Marseille Provence & Sainte Victoire & PACA (FR) \\
\hline CETT-UB & - & - \\
\hline
\end{tabular}

Fuente: Elaboración propia a partir de la documentación del proyecto.

El objetivo del proyecto "EMbleMatiC" fue la creación y la puesta en marcha de una oferta turística basada en la valorización de los activos territoriales de entornos montañosos cercanos al litoral mediterráneo. Dicha oferta, que debía basarse en los principios del turismo sostenible y responsable, aspiraba a constituir una alternativa al turismo de masas, tanto de sol y playa como de alta montaña. En la práctica, el proyecto perseguía tres objetivos estratégicos: (i) equilibrar los flujos turísticos entre el mar y la montaña mediante la desestacionalización de la oferta y el incremento del retorno económico para las áreas de interior; (ii) construir de forma participada una oferta de turismo slow y ponerla en práctica con la creación de rutas ecoturísticas; (iii) incrementar el reconocimiento internacional de la red EMM mediante la creación y el uso de una marca común que reflejase la filosofía sostenible y responsable del producto.

El proyecto estaba formalmente dividido en tres fases: en la primera se encargó una investigación sobre las características comunes de todos los territorios participantes; en la segunda se desarrolló e implementó el producto turístico sobre el terreno; y en la tercera se transfirieron los resultados a otros territorios. Con todo ello, se pretendía operar como un laboratorio donde la cooperación transnacional llevase a la generación de capacidades (o capacity building) por parte de cada socio. Se actuó bajo el principio de ejemplaridad en cuanto a la gestión de los recursos territoriales propios, así como desde el convencimiento de los beneficios de la aproximación "de abajo a arriba", en la cual la población local debía jugar un papel fundamental. Por último, se persiguió fomentar nuevas formas de economía del turismo basadas en aspectos menos materiales y más vivenciales, en base al principal recurso de los territorios que forman parte de la red; es decir, los valores humanos e inmateriales.

El instrumento específico con el que los socios del proyecto EMbleMatiC llevaron a cabo las acciones mencionadas es lo que se convino en llamar ecojourney o - traducido al castellano- ecoviaje: un recorrido de turismo vivencial en base a itinerarios por los territorios 
representados en el proyecto. Cada uno de los recorridos fue diseñado y llevado a la práctica para contribuir a superar los retos comunes de la red, que se han mencionado más arriba.

\subsection{Objetivo y planteamiento del artículo}

En el presente artículo, nos proponemos presentar y describir el proceso de diseño y aplicación sobre el terreno de un método para crear itinerarios turísticos como producto dinamizador de territorios de montaña en los que apenas repercuten los beneficios de la actividad turística costera. Como hemos presentado en el epígrafe anterior, dicho proceso se ha llevado a cabo en nueve territorios distribuidos a lo largo de la vertiente norte del Mediterráneo. En otras palabras, en este artículo presentamos nuestra experiencia, como socio académico de la red EMM, de los esfuerzos de la misma por contribuir al desarrollo local, a través del turismo sostenible y responsable, de las regiones por ella representada.

El fin último de este artículo es, por un lado, el de contribuir al debate académico acerca de cómo el ecoturismo puede ser una aportación positiva para la gestión territorial en ámbitos de montaña. Por otro lado, con los resultados del proceso aquí descrito pretendemos mostrar el potencial del método diseñado y aplicado para otros ámbitos territoriales que afronten retos similares a los de los miembros de la red EMM.

Este artículo tiene un carácter eminentemente aplicado, pues expone el planteamiento y los resultados de la puesta en práctica de los ecoviajes como instrumento de dinamización territorial. Tras unas breves notas de carácter teórico, el segundo capítulo describe al detalle el proceso interno que desembocó en el diseño del método utilizado, basado en 30 atributos que todo itinerario debe cumplir para convertirse en un ecoviaje. El tercer capítulo expone los resultados de la puesta en práctica de los ecoviajes en los nueve ámbitos (o destinos turísticos) que participaron en el proyecto EMbleMatiC. Es decir, cómo los socios del proyecto abordaron los 30 atributos y qué dificultades encontraron para cumplirlos. Finalmente, el capítulo 4 recapitula todo aquello expuestos en los capítulos 2 y 3 .

\subsection{Turismo y sostenibilidad: una breve aproximación teórica}

Pese a que los limites conceptuales entre turismo sostenible y turismo responsable son borrosos (Idelhadj, Rivera y Rodríguez, 2012; Cañizares, 2013), se entiende como turismo sostenible aquel que satisface las necesidades presentes de los visitantes y el territorio, protegiendo la capacidad de responder a las necesidades económicas distribuyendo equitativamente los beneficios; las socioculturales, asegurando la igualdad y la calidad de vida de la comunidad local; y respetando la diversidad medioambiental y los procesos ecológicos, en un intento de equilibrar la conservación y explotación de recursos (World Commission on Environment and Development [WCED], 1987; Organización Mundial de Turismo [OMT], 1999).

Según Ávila Bercial y Barrado Timón (2005), sin embargo, el turismo sostenible no solo implica la preservación de recursos, sino que es fundamental para la conformación de destinos referentes en términos de calidad. De hecho, son varios los autores que defienden la multidimensionalidad del concepto de la sostenibilidad (López Palomeque y Torres Delgado, 2011), respondiendo a un fenómeno social en que los criterios de ecología y responsabilidad pasan a formar parte de la experiencia turística. 
Por otra parte, el turismo responsable se entiende como una forma de hacer turismo que se rige por principios éticos y de responsabilidad social, además de por los parámetros más convencionales del turismo sostenible, siendo también respetuosa con los recursos naturales y los rasgos culturales de la sociedad. De esta manera, el turismo responsable pretende promover el comercio justo, el turismo comunitario orientado a la participación de las poblaciones anfitrionas en su desarrollo como principales beneficiarias de los ingresos por turismo, la igualdad y perspectiva de género o el "turismo accesible" y "para todos" (García, 2010; Lanquar y Rivera, 2010; Rivera y Rodríguez, 2012; Cañizares, 2013).

Adicionalmente a estos términos, se han añadido a la etimología turística conceptos como ecoturismo o turismo slow, dando lugar a una serie de etiquetas que definen la realización de visitas turísticas en contacto con la naturaleza. Ambos serán tratados a continuación.

El neologismo de "ecoturismo", objeto de definición en aumento desde el nuevo milenio (Castellanos y Orgaz, 2013), se define como una modalidad de turismo desarrollada particularmente en áreas naturales, de importancia ecológica, con el fin de apreciar sus atractivos paisajísticos y culturales, promoviendo un bajo impacto ambiental y propiciando la implicación activa de las poblaciones locales y su desarrollo local sostenible (CeballosLascurain, 1992; Mulero y Rivera, 2018). Además, según la OMT (2002), el ecoturismo promueve la conservación y la preservación tanto por parte de los visitantes como por empresas y stakeholders o actores locales, buscando el desarrollo de la actividad con la mínima producción de impactos negativos mediante elementos de interpretación y educación.

Finalmente, el turismo slow, como otras nuevas formas de hacer turismo, no goza de un consenso general sobre su definición, lo que dificulta identificar modelos para su desarrollo (Assaf y Baloglu, 2016). Sin embargo, si se coincide en su origen, siendo una mentalidad y movimiento nacido para escapar del ritmo de vida acelerado (Matos, 2004) y surgido como alternativa al turismo y consumo de masas para combinar la lentitud, la calidad de vida y la exploración, siendo a la vez una forma de hacer turismo y motor de cambio de la industria.

El panorama etimológico propone, por tanto, un contexto confuso, con conceptualizaciones complejas, en que turismo sostenible no responde a una única modalidad, sino a un paraguas amplio de actuación, una ideología común en la que, ante la tendencia a oponerse al turismo de masas, se adapta a las circunstancias del territorio (Pérez, 2004), difiriendo en el valor otorgado a los valores de la comunidad y el medio natural y el papel que estos adquieren en la relación entre turista y comunidad (Mulero y Rivera, 2018).

\section{El método: Los Términos de Referencia (TR) de los itinerarios.}

Un proyecto colectivo implica unos objetivos y unos métodos que puedan ser compartidos por todos los integrantes. Con una idea bien definida desde un primer momento (el instrumento debían ser rutas ecoturísticas), el consorcio inició un proceso de discusión sobre cómo garantizar que los itinerarios compartieran una serie de características que reflejaran el carácter de la red EMM (ver apartado 1). Y lo que es más importante: que dichos itinerarios pudiesen ser promocionados, con posterioridad, como un solo producto.

El proceso se inició de forma anterior a la definición de las rutas que cada socio quería ofrecer, pero con la base previa de los resultados de la primera fase del proyecto: un informe detallado acerca de las características comunes de los nueve territorios que componen la red EMM. De este modo, la primera consulta a los socios consistió en un cuestionario con 18 preguntas cerradas (a responder con un "sí" o un "no") acerca de las características y servicios 
que cada socio estaba en condiciones de dotar para su ruta propuesta. Este cuestionario (Tabla 2) fue el embrión de los posteriores Términos de Referencia (TR).

Tabla 2: Cuestionario de la consulta previa al diseño de los TR.

\begin{tabular}{|l|l|}
\hline $\mathbf{N}^{\mathbf{~}}$ & \multicolumn{1}{|c|}{ Pregunta } \\
\hline 1 & ¿Optimiza el itinerario las infraestructuras o inversiones existentes? \\
\hline 2 & ¿Fomenta el itinerario modos de transporte sostenibles? \\
\hline 3 & ¿Contribuye el itinerario de forma activa a la preservación de la biodiversidad? \\
\hline 4 & ¿Incluye el itinerario elementos de patrimonio histórico y cultural? \\
\hline 5 & $\begin{array}{l}\text { ¿Incluye el itinerario buenas prácticas socioeconómicas (por ej. integrando a } \\
\text { personas en riesgo de exclusión o adoptando los principios de la economía social } \\
\text { y solidaria)? }\end{array}$ \\
\hline 6 & $\begin{array}{l}\text { ¿Realizan los gestores del itinerario un seguimiento sobre los impactos de las } \\
\text { visitas? }\end{array}$ \\
\hline 7 & $\begin{array}{l}\text { ¿Incluye el itinerario elementos intangibles que muestren el carácter vivo del } \\
\text { territorio? }\end{array}$ \\
\hline 8 & ¿Incluye el itinerario guía e interpretación? \\
\hline 9 & $\begin{array}{l}\text { ¿Incluye el itinerario propuestas relacionadas con la producción y la gastronomía } \\
\text { locales? }\end{array}$ \\
\hline 10 & ¿Ofrece el itinerario un acceso gratuito? \\
\hline 11 & $\begin{array}{l}\text { ¿Es el visitante algo más que un simple consumidor en el itinerario? ¿Tienen los } \\
\text { visitantes posibilidades de conocer a la población local? }\end{array}$ \\
\hline 12 & ¿Están los actores locales involucrados en el proyecto de itinerario? \\
\hline 13 & ¿Minimiza el itinerario el riesgo de sufrir lesiones o la inseguridad? \\
\hline 14 & ¿Cuenta el itinerario con alojamientos con alguna certificación ambiental? \\
\hline 15 & $\begin{array}{l}\text { ¿Cuenta el itinerario con servicios gastronómicos que posean alguna certificación } \\
\text { (p. ej. slow food, km 0, comida ecológica, comercio justo...)? }\end{array}$ \\
\hline 16 & ¿Ofrece el itinerario la posibilidad de conocer productores locales? \\
\hline 17 & $\begin{array}{l}\text { ¿Ofrece el itinerario actividades de ocio que respeten el entorno (además de rutas } \\
\text { a pie)? }\end{array}$ \\
\hline 18 & $\begin{array}{l}\text { ¿Cuenta el itinerario con recursos culturales que posean alguna certificación } \\
\text { ambiental? }\end{array}$ \\
\hline
\end{tabular}

Fuente: Elaboración propia.

Con los resultados de dicha consulta en la mano (ver apartado 3, Tabla 9), se elaboró una lista de 30 atributos asociados a cinco temáticas diferentes; temáticas que vienen a cubrir la práctica totalidad de los aspectos a tener en cuenta cuando se crea un producto turístico de estas características. A continuación, presentamos los atributos, acompañados por una serie de materiales pensados para proporcionar evidencia de su cumplimiento. Dichos materiales son una pieza fundamental para controlar el proceso de diseño y ejecución de un ecoviaje. Los atributos se presentan agrupados en cada uno de los mencionados ejes temáticos que se exponen a continuación.

- Características básicas del itinerario: son atributos fundamentales y que marcan el carácter de las rutas, como su longitud y dificultad, pero también su accesibilidad. Además, un ecoviaje 
debe basarse en el aprovechamiento de las infraestructuras existentes y en el hecho de evitar puntos particularmente saturados por flujos turísticos (Tabla 3).

Tabla 3: Atributos relativos a las características básicas de los itinerarios.

\begin{tabular}{|c|c|c|}
\hline $\begin{array}{l}\text { Nodd } \\
\text { atributo }\end{array}$ & Nombre del atributo & Evidencias asociadas \\
\hline 1 & Localizado en el piedemonte & \multirow{2}{*}{ Mapa y descripción del itinerario } \\
\hline 2 & Grado de dificultad bajo a medio & \\
\hline 3 & $\begin{array}{l}\text { Accesible durante todo el año } \\
\text { (exceptuando episodios } \\
\text { meteorológicos extremos) }\end{array}$ & Calendario de apertura \\
\hline 4 & $\begin{array}{l}\text { Señalizado de manera apropiada (con } \\
\text { señalización física o digital) }\end{array}$ & Proyecto de señalización \\
\hline 5 & $\begin{array}{l}\text { Incluye puntos panorámicos } \\
\text { señalizados adecuadamente }\end{array}$ & $\begin{array}{l}\text { Mapa del itinerario y descripción de } \\
\text { los puntos seleccionados }\end{array}$ \\
\hline 6 & Favorece puntos no saturados & $\begin{array}{l}\text { Mapa y descripción del itinerario que } \\
\text { explique cómo el trayecto evita puntos } \\
\text { saturados }\end{array}$ \\
\hline 7 & Optimiza la infraestructura existente & Catálogo de elementos existentes \\
\hline
\end{tabular}

Fuente: Elaboración propia.

- Servicios asociados al itinerario: se refieren a los servicios y equipamientos que el visitante encontrará en su recorrido, desde alojamiento hasta elementos de interpretación del paisaje, pasando por oficinas turísticas, señalización o actividades ofertadas (Tabla 4).

Tabla 4: Atributos relativos a los servicios asociados de los itinerarios.

\begin{tabular}{|c|c|c|}
\hline $\begin{array}{l}\text { Nodd } \\
\text { atributo }\end{array}$ & Nombre del atributo & Evidencias asociadas \\
\hline 8 & $\begin{array}{l}\text { Incluye servicios/equipamientos de } \\
\text { interpretación ambiental/paisajística }\end{array}$ & $\begin{array}{l}\text { Breve descripción de los } \\
\text { servicios/equipamientos existentes }\end{array}$ \\
\hline 9 & $\begin{array}{l}\text { Incluye visitas al patrimonio cultural } \\
\text { y monumentos }\end{array}$ & \multirow{2}{*}{$\begin{array}{l}\text { Breve descripción de las actividades } \\
\text { planificadas }\end{array}$} \\
\hline 10 & $\begin{array}{l}\text { Incluye contenidos relacionados con } \\
\text { el patrimonio intangible }\end{array}$ & \\
\hline 11 & Ofrece servicios de guías locales & $\begin{array}{l}\text { Lista de los servicios de guía que } \\
\text { incluya su relación con el territorio }\end{array}$ \\
\hline 12 & $\begin{array}{l}\text { Ofrece actividades de ocio } \\
\text { respetuosas con el entorno (por ej. } \\
\text { deporte, salud y bienestar...) }\end{array}$ & Lista de actividades opcionales \\
\hline 13 & Ofrece gastronomía local & $\begin{array}{l}\text { Lista de establecimientos y sus } \\
\text { certificaciones (por ej. Slow food, Km } \\
0, \text { ecológico, comercio justo...) }\end{array}$ \\
\hline
\end{tabular}




\begin{tabular}{|l|l|l|}
\hline 14 & $\begin{array}{l}\text { Ofrece la posibilidad de conocer a } \\
\text { productores locales y de adquirir sus } \\
\text { productos }\end{array}$ & $\begin{array}{l}\text { Lista de productos locales y de su } \\
\text { relación con el territorio }\end{array}$ \\
\hline 15 & $\begin{array}{l}\text { Si ofrece servicio de picnic, este debe } \\
\text { ser sostenible }\end{array}$ & Guías de picnic para establecimientos \\
\hline
\end{tabular}

Fuente: Elaboración propia.

- Sostenibilidad y aspectos éticos: un ecoviaje se distingue de otros productos por cumplir con una serie de criterios relacionados con la sostenibilidad ambiental (e.g. movilidad sostenible), social (por ej. interacción con la población local) y económica (por ej. buenas prácticas ambientales por parte de los establecimientos asociados al recorrido) (Tabla 5).

Tabla 5: Atributos relativos a la sostenibilidad y aspectos éticos de los itinerarios.

\begin{tabular}{|c|c|c|}
\hline $\begin{array}{c}\mathbf{N}^{0} \text { del } \\
\text { atributo }\end{array}$ & Nombre del atributo & Evidencias asociadas \\
\hline 16 & $\begin{array}{l}\text { Ofrece la opción de llegar en } \\
\text { transporte público }\end{array}$ & $\begin{array}{l}\text { Descripción de las posibilidades } \\
\text { existentes de transporte público }\end{array}$ \\
\hline $16 b$ & $\begin{array}{l}\text { Ofrece estímulos para que los } \\
\text { visitantes compensen sus emisiones } \\
\text { de gases de efecto invernadero }\end{array}$ & $\begin{array}{l}\text { Información para visitantes sobre } \\
\text { cómo pueden compensar su huella de } \\
\text { carbono }\end{array}$ \\
\hline 17 & $\begin{array}{l}\text { Promueve la movilidad sostenible } \\
\text { dentro del itinerario }\end{array}$ & $\begin{array}{l}\text { Mapa y descripción del itinerario. Si el } \\
\text { itinerario permite circulación } \\
\text { motorizada, se deben detallar las } \\
\text { medidas previstas para fomentar la } \\
\text { movilidad sostenible }\end{array}$ \\
\hline 18 & $\begin{array}{l}\text { Promueve la interacción entre } \\
\text { visitantes y población local }\end{array}$ & $\begin{array}{l}\text { Breve descripción de las posibles } \\
\text { interacciones (por ej. a través de } \\
\text { alojamientos, actividades de ocio...) }\end{array}$ \\
\hline 19 & $\begin{array}{l}\text { Prioriza alojamientos gestionados por } \\
\text { actores locales (por ej. a través de } \\
\text { acuerdos locales de calidad) }\end{array}$ & $\begin{array}{l}\text { Lista de establecimientos a incluir en } \\
\text { una guía turística }\end{array}$ \\
\hline 20 & $\begin{array}{l}\text { Proporciona información clara y } \\
\text { visual sobre el comportamiento } \\
\text { adecuado de los visitantes }\end{array}$ & Normas para visitantes \\
\hline 21 & $\begin{array}{l}\text { Fomenta la concienciación sobre } \\
\text { prácticas de turismo responsable entre } \\
\text { los actores locales }\end{array}$ & $\begin{array}{l}\text { Directrices de buenas prácticas } \\
\text { ambientales }\end{array}$ \\
\hline
\end{tabular}

Fuente: Elaboración propia.

- Gobernanza y gestión: un ecoviaje debe ser sostenible en tanto que producto turístico. Ello se consigue aplicando una serie de herramientas de control interno y de seguimiento a largo plazo, que son recogidas en los atributos aquí incluidos (Tabla 6). 
Tabla 6: Atributos relativos a la gobernanza y la gestión de los itinerarios.

\begin{tabular}{|l|l|l|}
\hline $\begin{array}{l}\mathbf{N}^{\mathbf{0}} \text { del } \\
\text { atributo }\end{array}$ & Nombre del atributo & Evidencias asociadas \\
\hline 22 & $\begin{array}{l}\text { Aplica herramientas de seguimiento } \\
\text { para mejorar la sostenibilidad de la } \\
\text { destinación }\end{array}$ & Plan de acción \\
\hline 23 & Integra a actores locales en su diseño & $\begin{array}{l}\text { Base de datos de actores locales que } \\
\text { incluya su papel en el proceso de } \\
\text { diseño }\end{array}$ \\
\hline 24 & $\begin{array}{l}\text { Utiliza indicadores para monitorear y } \\
\text { gestionar su impacto territorial }\end{array}$ & Lista de indicadores a utilizar \\
\hline 25 & $\begin{array}{l}\text { Comparte experiencias de forma } \\
\text { periódica con otros miembros de la } \\
\text { red EMM }\end{array}$ & $\begin{array}{l}\text { Calendario de encuentros y de } \\
\text { comunicación periódica }\end{array}$ \\
\hline 26 & $\begin{array}{l}\text { Proporciona material interpretativo en } \\
\text { las lenguas locales y extranjeras } \\
\text { (inglés) }\end{array}$ & $\begin{array}{l}\text { Lista de materiales a producir, en } \\
\text { lenguas locales e inglés }\end{array}$ \\
\hline
\end{tabular}

Fuente: Elaboración propia.

- Marketing: un ecoviaje no es más que la marca turística de una ruta por un territorio. Por ello, para poder dar salida al producto en el mercado, son necesarios elementos de marketing ecoturístico (Tabla 7).

Tabla 7: Atributos relativos al marketing de los itinerarios.

\begin{tabular}{|l|l|l|}
\hline $\begin{array}{c}\text { No del } \\
\text { atributo }\end{array}$ & \multicolumn{1}{|c|}{ Nombre del atributo } & Evidencias asociadas \\
\hline 27 & $\begin{array}{l}\text { Informa acerca de los servicios disponibles en la } \\
\text { ruta }\end{array}$ & $\begin{array}{l}\text { Lista de servicios a } \\
\text { incluir en una guía } \\
\text { turística }\end{array}$ \\
\hline 28 & $\begin{array}{l}\text { Orientado tanto a visitantes locales como a } \\
\text { visitantes procedentes de otros lugares } \\
\text { (preferentemente de la costa), ofreciendo } \\
\text { actividades complementarias o alternativas }\end{array}$ & \multirow{2}{*}{ Estrategia de marketing } \\
\cline { 1 - 2 } 29 & $\begin{array}{l}\text { Orientado a un perfil de visitante común para la red } \\
\text { Promueve una marca y una estrategia de }\end{array}$ & \\
\hline 30 & márqueting comunes & \\
\hline
\end{tabular}

Fuente: Elaboración propia.

Lo que se acaba de presentar es la versión final de los TR adoptada por el consorcio. Sin embargo, hasta llegar a dicha versión se produjo un proceso de discusión con todos los socios. El primer paso fue enviar los TR a cada uno de los socios para que ellos mismos autoevaluaran su capacidad para abordar cada uno de los atributos. Los resultados de esta consulta fueron fundamentales para (i) reestructurar la lista de atributos (con ligeros cambios relativos a su contenido y exigencia), y (ii) detectar qué atributos presentaban mayores dificultades y/o reticencias por parte de los socios. Una reunión plenaria (convocada ad-hoc para resolver este 
asunto) sirvió para encontrar el deseado consenso en todos y cada uno de los atributos. Fue necesario aclarar al detalle el contenido requerido en cada atributo (reflejado en las evidencias) para poder llegar a la versión final. Es importante aclarar que, para aquellos atributos más "problemáticos", desde el CETT nos encargamos de proveer todos aquellos materiales necesarios para que los socios trabajasen sobre una base común, que les ayudase a afrontar los retos que dichos atributos les suponía. La tabla 8 muestra dichos atributos, así como los materiales facilitados por el CETT para cada uno de ellos.

Tabla 8: Atributos con materiales de soporte proporcionados por el CETT.

\begin{tabular}{|c|c|c|}
\hline $\begin{array}{c}\mathbf{N}^{0} \text { del } \\
\text { atributo }\end{array}$ & Nombre del atributo & Material facilitado por el CETT \\
\hline 13 & Ofrece gastronomía local & \multirow[b]{2}{*}{$\begin{array}{l}\text { Plantilla de tabla con las siguientes } \\
\text { categorías: nombre, contacto, } \\
\text { certificaciones, grado de implicación en el } \\
\text { proyecto (dividido en tres categorías: } \\
\text { "implicado", "potencialmente implicado", } \\
\text { "no implicado") }\end{array}$} \\
\hline 14 & $\begin{array}{l}\text { Ofrece la posibilidad de conocer } \\
\text { productores locales y de adquirir } \\
\text { sus productos }\end{array}$ & \\
\hline 15 & $\begin{array}{l}\text { Si ofrece servicio de picnic, este } \\
\text { debe ser sostenible }\end{array}$ & $\begin{array}{l}\text { Breves directrices sobre los aspectos que } \\
\text { deben tenerse en cuenta a la hora de ofrecer } \\
\text { servicios de picnic en el ecoviaje. }\end{array}$ \\
\hline $16 b$ & $\begin{array}{l}\text { Ofrece estímulos para que los } \\
\text { visitantes compensen sus } \\
\text { emisiones de gases de efecto } \\
\text { invernadero }\end{array}$ & $\begin{array}{l}\text { Hoja informativa (para distribuir entre los } \\
\text { agentes locales implicados) acerca de cómo } \\
\text { funciona la compensación de la huella de } \\
\text { carbono.Incluye el enlace a una plataforma } \\
\text { de compensación de emisiones. }\end{array}$ \\
\hline 20 & $\begin{array}{l}\text { Proporciona información clara y } \\
\text { visual sobre el comportamiento } \\
\text { adecuado de los visitantes }\end{array}$ & $\begin{array}{l}\text { Dos propuestas con caracteres diferentes: } \\
\text { una más clásica, con las medidas } \\
\text { redactadas en un tono coercitivo; otra más } \\
\text { creativa, con medidas redactadas en un } \\
\text { tono propositivo. }\end{array}$ \\
\hline 21 & $\begin{array}{l}\text { Fomenta la concienciación sobre } \\
\text { prácticas de turismo responsable } \\
\text { entre los visitantes }\end{array}$ & $\begin{array}{l}\text { "Decálogo" con nueve medidas concretas a } \\
\text { adoptar, que los socios deben dar a conocer } \\
\text { a los agentes locales implicados. El } \\
\text { documento incluye un enlace a un } \\
\text { exhaustivo documento publicado por la } \\
\text { Comisión Europea (Styles et al., 2013), que } \\
\text { puede ser utilizado por los actores locales } \\
\text { como herramienta práctica. }\end{array}$ \\
\hline 22 & $\begin{array}{l}\text { Aplica herramientas de } \\
\text { seguimiento para mejorar la } \\
\text { sostenibilidad de la destinación }\end{array}$ & $\begin{array}{l}\text { Plantilla de seguimiento del plan de acción, } \\
\text { que incluye el nombre del objetivo } \\
\text { perseguido por cada medida, el grado de } \\
\text { prioridad, el agente responsable de su } \\
\text { ejecución, el presupuesto aproximado, el } \\
\text { plazo para su ejecución y su seguimiento, } \\
\text { la descripción concreta de la medida a } \\
\text { efectuar, el indicador que permite }\end{array}$ \\
\hline
\end{tabular}




\begin{tabular}{|c|c|c|}
\hline & & $\begin{array}{l}\text { comprobar la evolución, y la comprobación } \\
\text { final de la consecución de la medida. }\end{array}$ \\
\hline 23 & $\begin{array}{l}\text { Integra a actores locales en su } \\
\text { diseño }\end{array}$ & $\begin{array}{l}\text { Plantilla de tabla con las siguientes } \\
\text { categorías: nombre del actor, contacto, } \\
\text { localización geográfica, campo/temática, } \\
\text { papel a jugar en el proyecto, ámbito de } \\
\text { intervención. }\end{array}$ \\
\hline 24 & $\begin{array}{l}\text { Utiliza indicadores para } \\
\text { monitorear y gestionar su impacto } \\
\text { territorial }\end{array}$ & $\begin{array}{l}\text { Propuesta de diez indicadores } \\
\text { seleccionados del ETIS (European Tourism } \\
\text { Information System) (Comisión Europea, } \\
\text { 2017) }\end{array}$ \\
\hline 25 & $\begin{array}{l}\text { Comparte experiencias de forma } \\
\text { periódica con otros miembros de } \\
\text { la red EMM }\end{array}$ & $\begin{array}{l}\text { Propuesta esquemática para mantener el } \\
\text { contacto regular entre socios de la red } \\
\text { EMM tras la finalización del proyecto } \\
\text { EMbleMatiC. }\end{array}$ \\
\hline 28 & $\begin{array}{l}\text { Orientado tanto a visitantes } \\
\text { locales como a visitantes } \\
\text { procedentes del litoral, ofreciendo } \\
\text { una actividad alternativa }\end{array}$ & \multirow{3}{*}{ Estrategia de márqueting (marketing brief) } \\
\hline 29 & $\begin{array}{l}\text { Orientado a un perfil de visitante } \\
\text { común para la red }\end{array}$ & \\
\hline 30 & $\begin{array}{l}\text { Promueve una marca y una } \\
\text { estrategia de márqueting comunes }\end{array}$ & \\
\hline
\end{tabular}

Fuente: Elaboración propia.

\section{Resultados y Discusión}

Tal y como hemos detallado en el apartado 2, el proceso de creación de los TR se inició con una consulta en base a 18 preguntas cerradas. Los resultados de la misma se reflejan en la tabla 9, idéntica a la tabla 2, pero esta vez con las preguntas clasificadas en tres clases cromáticas según las respuestas de los socios Las preguntas resaltadas en color verde (5 de 18) fueron respondidas positivamente por todos los socios. Las preguntas en color amarillo fueron respondidas positivamente por todos los socios, excepto por uno de ellos. En el caso de las preguntas en color calabaza, las respuestas negativas ascendieron a dos. Por último, las preguntas resaltadas en color rojo fueron respondidas negativamente por la mayoría de los socios. Nótese que, en la gran mayoría de preguntas (13 de 18), todos los socios (o una gran mayoría de ellos) dicen contar con lo que se les demanda. Hay, sin embargo, cinco aspectos (los resaltados en rojo) que dejan claro que representan un obstáculo importante para todos o casi todos los socios. 
Tabla 9: Cuestionario de la consulta previa al diseño de los TR, con los resultados en una escala cromática.

\begin{tabular}{|c|c|}
\hline $\mathbf{N}^{\mathbf{0}}$ & Pregunta \\
\hline 1 & ¿Optimiza el itinerario las infraestructuras o inversiones existentes? \\
\hline 2 & ¿Fomenta el itinerario modos de transporte sostenibles? \\
\hline 3 & ¿Contribuye el itinerario de forma activa a la preservación de la biodiversidad? \\
\hline 4 & ¿Incluye el itinerario elementos de patrimonio histórico y cultural? \\
\hline 5 & $\begin{array}{l}\text { ¿Incluye el itinerario buenas prácticas socioeconómicas (por ej. integrando a } \\
\text { personas en riesgo de exclusión o adoptando los principios de la economía } \\
\text { social y solidaria)? }\end{array}$ \\
\hline 6 & $\begin{array}{l}\text { ¿Realizan los gestores del itinerario un seguimiento sobre los impactos de las } \\
\text { visitas? }\end{array}$ \\
\hline 7 & $\begin{array}{l}\text { ¿Incluye el itinerario elementos intangibles que muestren el carácter vivo del } \\
\text { territorio? }\end{array}$ \\
\hline 8 & ¿Incluye el itinerario guía e interpretación? \\
\hline 9 & $\begin{array}{l}\text { ¿Incluye el itinerario propuestas relacionadas con la producción y la } \\
\text { gastronomía locales? }\end{array}$ \\
\hline 10 & ¿Ofrece el itinerario un acceso gratuito? \\
\hline 11 & $\begin{array}{l}\text { ¿Es el visitante algo más que un simple consumidor en el itinerario? ¿Tienen } \\
\text { los visitantes posibilidades de conocer a la población local? }\end{array}$ \\
\hline 12 & ¿Están los actores locales involucrados en el proyecto de itinerario? \\
\hline 13 & ¿Minimiza el itinerario el riesgo de sufrir lesiones o la inseguridad? \\
\hline 14 & ¿Cuenta el itinerario con alojamientos con alguna certificación ambiental? \\
\hline 15 & $\begin{array}{l}\text { ¿Cuenta el itinerario con servicios gastronómicos que posean alguna } \\
\text { certificación (por ej. slow food, } \mathrm{km} 0 \text {, comida ecológica, comercio justo...)? }\end{array}$ \\
\hline 16 & ¿Ofrece el itinerario la posibilidad de conocer productores locales? \\
\hline 17 & $\begin{array}{l}\text { ¿Ofrece el itinerario actividades de ocio que respeten el entorno (además de } \\
\text { rutas a pie)? }\end{array}$ \\
\hline 18 & $\begin{array}{l}\text { ¿Cuenta el itinerario con recursos culturales que posean alguna certificación } \\
\text { ambiental? }\end{array}$ \\
\hline
\end{tabular}

Fuente: Elaboración propia.

Una vez establecida la versión final de los TR (Tablas 3 a 7), iniciamos un proceso de seguimiento del nivel de cumplimiento de los atributos. Dicho proceso fue exhaustivo y sistemático, prologándose durante algo más de un año. A efectos prácticos, se configuró una sencilla tabla de seguimiento a través de la cual cada socio disponía de información individual acerca del grado de cumplimiento de cada atributo. La comunicación bilateral (entre cada socio y el CETT), así como multilateral (entre todos los socios) facilitó el proceso. A continuación, exponemos los resultados de dicho proceso de seguimiento. Hemos estructurado la exposición en tres grandes ámbitos temáticos: trabajo en red (3.1), participación de los actores locales (3.2) y sostenibilidad (3.3). Nuestra intención es hacer más visibles las dificultades encontradas y las lecciones obtenidas.

Los contenidos de este capítulo están basados en dos documentos que resultaron del propio proceso de monitoreo: por un lado, un informe experimental (Experimentation Report Autores, 2019a), muy exhaustivo, que derivó de un proceso de consulta interno con los socios del proyecto, basado en un cuestionario individual y en una discusión grupal. Por el otro, una guía de transferencia (Sancho y Serrat, 2019b), en la que se recogen las experiencias y se presentan a un público externo en forma de lecciones aprendidas. 


\subsection{La complejidad del trabajo en red}

Como hemos descrito anteriormente, el proyecto EMbleMatiC fue un intento de llevar a nueve entidades que representan nueve realidades diferentes a un terreno común, en base a la identificación de unos retos a afrontar y, sobre todo, en base a unas estrategias e instrumentos (el ecoviaje) que permitan afrontar dichos retos. El proyecto descansaba en la filosofía del trabajo en red; es decir: el intercambio y el apoyo mutuo para la consecución colectiva de los objetivos marcados. Tras tres años de trabajo, llegamos a la conclusión de que el elemento clave del éxito es la búsqueda satisfactoria de puntos de acuerdo consensual (o muy cercanos) al consenso. Durante el proyecto, hemos utilizado sistemáticamente el símil matemático del mínimo común denominador: para poder avanzar todos juntos (y no de forma individual y, por tanto, desigual), es fundamental establecer una base de mínimo acuerdo y comprometerse a seguirla. Esto es lo que representa los TR con sus 30 atributos.

Naturalmente, la búsqueda y el mantenimiento de un denominador común son una tarea ardua y que requiere altas dosis de rigor y, al mismo tiempo, de capacidad para ceder y acordar. Recuérdese que la red cuenta con nueve miembros que, pese a compartir problemáticas comunes, difieren en muchos aspectos que determinan su capacidad de influencia sobre el terreno:

- En primer lugar, el carácter jurídico de cada entidad, que marca aquello que puede o debe realizar: por ejemplo, la potestad o no de instalar señalización (atributo $n^{\circ} 4$ ) u otros elementos de interpretación paisajística (atributo $\mathrm{n}^{\circ}$ 5). Del mismo modo, el carácter jurídico influye en la capacidad de movilización de los agentes locales.

- En segundo lugar, la propia situación interna de los socios. En algunos casos, existía una trayectoria y un trabajo previo que garantizaba una comunicación fluida con los actores territoriales. En el polo opuesto, nos hemos encontrado con entidades con poco arraigo territorial (debido a su carácter eminentemente administrativo y poco ligado a todo lo que representa el desarrollo local, o bien debido al contexto general de la región o el país por ej., no existencia o escaso peso de instrumentos de planeamiento y/o desarrollo turístico).

- En tercer lugar, la capacidad de adaptación de cada socio a la hoja de ruta común. Algunos socios tenían un proyecto bien definido de antemano, y dicho proyecto varió poco durante el proyecto EMbleMatiC. Si bien este hecho les dio una cierta ventaja en el proceso (por ejemplo, a la hora de establecer una red de actores locales o de ejecutar trabajo sobre el terreno), también existen casos en los que les restó flexibilidad; cosa que, a su vez, les comportó más dificultades para cumplir con algunos atributos. Dos ejemplos en este sentido son el $n^{\circ} 6$ (sobre la necesidad de evitar puntos saturados en el itinerario) y el $\mathrm{n}^{\mathrm{o}}$ 22 (sobre la aplicación de herramientas de seguimiento del proyecto), que será comentado al detalle a continuación.

Otros socios con un proyecto en un estadio menos desarrollado no necesariamente resultaron perjudicados. Al contrario: dicha situación se mostró ventajosa en el proceso de discusión sobre las características básicas de los ecoviajes. Por ejemplo, algunos socios modificaron las rutas seleccionadas en su territorio para facilitar el consenso acerca de los atributos básicos de los itinerarios (como, por ej., su localización en el piedemonte o la mejora de su accesibilidad).

Más allá del estadio de cada proyecto individual, también se produjeron situaciones en las que no se alcanzó un consenso "factual" alrededor de determinados atributos (que, 
consecuentemente, no fueron cumplidos por algunos socios). Nos referimos a aspectos sobre los cuales existían diferencias culturales, como el $n^{\circ} 15$ (sobre servicios de picnic sostenibles, que será abordado más adelante), así como diferencias estratégicas relativas al tratamiento del alojamiento: en concreto, sobre si priorizar el alojamiento en manos de la población local (por ejemplo, incluyéndolo en la guía turística), tal y como rezan los atributos $\mathrm{n}^{\circ} 19$ y 27 . En estos casos, no todos los socios estuvieron dispuestos a proceder del modo que se consensuó inicialmente, pero ello no implicó la exclusión (ni siquiera la modificación) de dichos atributos.

El caso contrario lo encontramos en los atributos $n^{\circ} 8$ y 10 (sobre la inclusión de elementos interpretativos del paisaje y de elementos de patrimonio intangible, respectivamente). Si bien su contenido no fue objeto de discusión durante el proceso de redacción de los TR, y si bien su seguimiento mostró unas altas tasas de cumplimiento, el informe experimental posterior puso de manifiesto, sin embargo, que algunos socios percibieron su cumplimiento como "difícil" o, en algunos casos, "muy difícil".

Pero sin duda, el ejemplo que mejor ilustra la complejidad del trabajo en red es el desarrollo de la estrategia común de marketing (ver atributos 28 a 30, Tabla 7). La fase previa de dicha estrategia incluyó un proceso de consulta a los socios sobre el perfil de visitante que los ecoviajes deberían priorizar. El objetivo era la definición, lo más concreta posible, de perfiles basados en diferentes criterios demográficos y socioeconómicos. Las discusiones plenarias revelaron la enorme dificultad de consensuar un perfil común. Lo interesante fue que la razón principal no residía en la divergencia de prioridades (por ej. pensionistas versus jóvenes, o visitantes procedentes del propio país versus público internacional), sino en la voluntad unánime, por parte de los socios, de configurar un perfil muy abierto, en el que prácticamente cupiese todo el mundo. Esta posición, si bien podría ser interpretada como un logro de consenso, es cierto que dificulta la definición de la estrategia de márqueting: si no sabes a quién te diriges, resulta mucho más complicado definir el posicionamiento en el mercado y la estrategia de comunicación, incluyendo aspectos como la marca, el logo...

Además de lo expuesto hasta ahora, es importante destacar la dimensión local del trabajo en red. A nivel "doméstico", cada socio ha estado trabajando con su propia red de actores de naturaleza diversa: administraciones públicas, empresas de todo tipo (alojamientos, restauración, servicios de guías...), gestores de espacios protegidos, entidades de gestión del patrimonio material e inmaterial, ONGs, entidades de la sociedad civil. Este conjunto de actores forman una red en sí misma, y la función de cada socio era y es "tejer" dicha red para que los actores puedan actuar del modo más cooperativo posible. Una tarea extraordinariamente difícil, pues en la sociedad existen intereses contrapuestos y que, a menudo, tienden a colisionar cuando se trata de decidir actuaciones concretas en el territorio.

Los TR proporcionan herramientas relacionadas con la gestión y la gobernanza (Tabla 6) con las que abordar este reto mayúsculo. De todas ellas, queremos poner énfasis en el atributo $\mathrm{n}^{\mathbf{0}} 22$ y el instrumento propuesto por el CETT: el plan de acción (Tabla 8). Se trata de un documento en el que los socios debían pensar en medidas a ejecutar en el futuro inmediato en sus territorios; medidas destinadas a mejorar la gestión de los recursos turísticos. La redacción de los planes de acción por parte de los socios reflejó, una vez más, las desigualdades entre ellos. Mientras que algunos socios establecieron unas acciones concretas y detallaron plazos, agentes y recursos necesarios, otros tuvieron dificultades para definir sus acciones. Un escollo común (y esperado por nuestra parte) fue la definición de los recursos económicos necesarios para emprender la acción. En numerosos casos, tuvimos que insistir a los socios para que proporcionasen dicha información, aunque ella tuviera un carácter meramente indicativo. 
Una última dimensión del trabajo en red en el proyecto EMbleMatiC es la que tiene que ver con la llamada capacity building. Es decir, incrementar la capacidad de acción gracias a las sinergias que surgen del trabajo colaborativo. En este caso, el programa Interreg ofrece proyectos "horizontales", que tejen relaciones entre proyectos con similitud temática. Estos proyectos son fundamentales para alcanzar los objetivos estratégicos de la red EMM. A la práctica, para el proyecto EMbleMatiC, ello ha significado un esfuerzo para plasmar sobre el terreno los ecoviajes.

En resumen: la geometría variable que adopta el trabajo en red, según se trate del nivel del propio consorcio, del programa europeo de financiación de proyectos, o el nivel local (dentro de cada uno de los territorios), es lo que confiere un alto grado de complejidad a esta cuestión.

\subsection{El desigual grado de participación de los actores locales.}

De lo expuesto en la sección anterior se desprende que el grado de participación de los actores locales es decisivo para garantizar el enfoque "de abajo a arriba" en diseño del ecoviaje y, por ende, la viabilidad del proyecto. La principal conclusión derivada de la experiencia de aplicación de los TR es que dicho grado de participación ha sido muy desigual entre los socios del proyecto. Observamos que aquellos socios con mayor capacidad de movilización consiguieron involucrar a más actores y con mayor diversidad, mientras que aquellos actores más alejados de la realidad social y empresarial no consiguiera reunir una masa crítica que reflejase la diversidad social de su territorio. En este sentido, es importante puntualizar que cuando hablamos de integrar, no solamente nos referimos a movilizar, sino sobre todo a la capacidad de convencer a los actores para que se identifiquen con el proyecto y lo hagan suyo.

Un buen indicador del grado de implicación es el número de reuniones que cada socio celebró durante todo el recorrido del proyecto. Solamente dos de los nueve socios mantuvieron reuniones regulares con sus actores locales. El resto de socios tuvo un poder de convocatoria que, en la mayoría de los casos, es reseñable, aunque solo celebraron reuniones esporádicas; en algunos casos, incluso una sola vez al inicio del proyecto. Si bien durante el seguimiento del cumplimiento de los atributos no se cuantificaron las reuniones mantenidas, de la información aportada alrededor de los atributos $\mathrm{n}^{\circ}$ 13, 14 (Tabla 4) y 23 (Tabla 6) se deduce el comportamiento de cada socio. Las diferencias en la cantidad de locales de restauración y de productores locales incluidos en las evidencias, así como de actores en general, se observan a simple vista, y no solo desde el punto de vista cuantitativo, sino también cualitativo. A un nivel mucho más concreto, el atributo $\mathrm{n}^{\mathrm{o}} 11$ (sobre la incorporación de guías turísticos locales) también proporciona información interesante sobre las desigualdades entre los socios en este aspecto: mientras que, en algunos casos, este aspecto no supuso ningún problema, para otros socios fue una tarea difícil o muy difícil de alcanzar (como demuestran los resultados del informe experimental). Existen casos en los que los guías incorporados no pueden considerarse estrictamente locales.

Mención aparte merece el atributo $\mathrm{n}^{\mathrm{o}} 18$, sobre la necesidad de promover la interacción entre visitantes y población local. Más allá de su grado concreto de cumplimiento, el problema que encontramos aquí es la propia conceptualización del atributo. A pesar de los intentos propios y de la discusión con los socios, no fuimos capaces de definir de manera más concreta en qué deben (o pueden) consistir las interacciones entre visitantes y población local (más allá de las propias que propone - o dicta - la economía de mercado, donde se enmarca la actividad turística). El ejemplo que se mencionó más a menudo es el del alojamiento en hogares 
particulares. Sin duda, este es un punto que merecería debate profundo, tanto a nivel práctico como académico.

Si atendemos la opinión de los socios sobre este asunto, sorprende su percepción positiva. En el cuestionario empleado para redactar el informe experimental, todos los socios aseguraron haber involucrado a actores locales, y casi la mitad de ellos afirmaron que entre un tercio y la mitad de los actores contactados acabaron enrolándose en el proyecto. Todos los socios respondieron que el nivel de implicación de los actores había sido "alto" o "muy alto"). Bien es cierto que, en este mismo cuestionario, los socios aportaron una serie de factores que explican las dificultades encontradas, que resumimos a continuación:

- La estacionalidad propia del sector turístico, que provoca que muchos actores sean difíciles de contactar fuera de la temporada alta.

- La capacidad de selección de actores, que tiene que ver con el ya comentado carácter jurídico de la entidad.

- Las barreras legales o burocráticas, que recuerdan los límites y la necesidad de actuar en consonancia con las autoridades competentes en cada momento.

- La voluntad de los actores para formar parte del proyecto y sentirse parte responsable de su éxito o fracaso. A menudo se echa de menos la falta de voluntad, que se explica por la limitada disponibilidad temporal, pero también por el nivel de escepticismo en relación con la sostenibilidad del proyecto.

- La comunicación y la necesidad de "hablar el mismo idioma". A algunos actores del territorio les cuesta comprender determinados términos pertenecientes a la jerga propia de quien coordina proyectos de desarrollo turístico.

- El consumo de recursos temporales que representa, para la organización en cuestión, el trabajo individual previo de aproximación a cada actor.

El trabajo de los socios demuestra que estas dificultades pueden afrontarse si se establece una cultura de trabajo basada en reuniones regulares con un grupo de actores. Las reuniones grupales no son, sin embargo, incompatibles con encuentros bilaterales, especialmente cuando se desea ampliar la masa crítica de actores implicados en el proyecto.

\subsection{El tortuoso camino hacia la aplicación práctica de los principios de la sostenibilidad.}

Tanto durante el proceso de discusión para la conformación de la lista definitiva de atributos, como durante su aplicación sobre el terreno con los ecoviajes, se hizo patente la dificultad que supone afrontar los numerosos retos que comporta la sostenibilidad en su sentido más amplio.

Debemos recordar en este punto el principio del "mínimo común denominador". Existen ejemplos de atributos en los que dicho principio se aplicó de forma explícita ( $n^{\circ} 15$ - Tabla 4 , 19 y 21 -Tabla 5, 24 - Tabla 6, incluso el $\mathrm{n}^{\circ} 20$-Tabla 5). El caso del uso de indicadores para monitorear y gestionar su impacto territorial (atributo $\mathrm{n}^{\circ} 24$ ) es el más ilustrativo: mientras el CETT propuso una selección de criterios de la lista del ETIS (ver apartado 1), los socios insistieron en las enormes dificultades que afrontan para poder aplicar los indicadores (derivados, fundamentalmente, de la disponibilidad de datos y de la propia capacidad interna, a nivel de recursos humanos, de los socios). De hecho, la consulta previa a la redacción de los TR ya dejó entrever que este era uno de los aspectos donde los socios iban a encontrar mayores dificultades (Tabla 9). Y, efectivamente, así fue: este atributo fue calificado por los socios como 
el más difícil de afrontar de todo el proceso, según muestran los datos del informe experimental (Sancho y Serrat, 2019a). La solución "pactada" llegó tras una reducción drástica de los indicadores a aplicar. Finalmente, y con la perspectiva de todo el proyecto, ha quedado patente que esta cuestión deberá resolverse en el futuro, más allá del marco estricto del proyecto EMbleMatiC, tal y como argumentaremos más abajo.

En general, constatamos que aquellos temas más innovadores generaron un recelo inicial entre los socios, aunque a la postre fueron aceptados, al menos formalmente. El ejemplo más ilustrativo es la compensación de la huella de carbono (atributo $\mathrm{n}^{\circ} 16 \mathrm{~b}$ ). En este asunto, el CETT planteó la conveniencia de sensibilizar a los visitantes de todas las destinaciones acerca de las emisiones de gases de efecto invernadero que genera el transporte de grandes distancias. La primera reacción de los socios fue hostil: había, en general, desconocimiento sobre el funcionamiento de estos mecanismos, incluso sobre la idea en sí misma. Fue, pues, necesaria una labor pedagógica por nuestra parte: en primer lugar, insistiendo en el carácter estrictamente voluntario de los pagos por compensación (en un inicio, algunos socios estaban atemorizados por posibles nuevas "tasas"); posteriormente, presentando instrumentos concretos (es decir, plataformas online donde uno puede compensar sus propias emisiones) para que determinados actores implicados (alojamientos, pero también oficinas de información turística y agencias de viajes colaboradoras) difundieran entre sus visitantes (Tabla 8).

Más allá de los ejemplos concretos, es conveniente insistir en que la sostenibilidad del producto del ecoviaje dependerá del modo en que la red EMM (y cada miembro de forma individual) afronte el medio y el largo plazo. Es ahí donde los TR deben contribuir de forma decisiva: su cumplimiento y seguimiento deben ir más allá del proyecto EMbleMatiC, pese a la incertidumbre ligada a la financiación.

En resumen, queda por ver el grado de compromiso con estos aspectos por parte de los socios; compromiso que debería traducirse en una actitud proactiva trabajando por la concienciación, tanto de los visitantes (en el caso de la compensación de la huella de carbono) como de los actores locales (en el caso de las certificaciones y las buenas prácticas ambientales).

\section{Conclusiones}

Si comparamos los resultados de la consulta previa a la redacción de los TR (Tabla 9) con los resultados del proceso de seguimiento del cumplimiento de los 30 atributos (expuesto en las secciones 3.1, 3.2 y 3.3), observamos numerosos paralelismos que, para nosotros, distan de ser casuales. Si bien constatamos el progreso efectuado por todos los socios (progreso que, en algunos casos, ha sido realmente positivo), la conclusión principal es que los temas que se antojaban difíciles al inicio del proceso, antes de redactar los TR, son los que han resultado de mayor dificultad. Son temas que requieren un esfuerzo que va más allá de la forma habitual de gestionar recursos turísticos (lo que los anglosajones denominan business as usual). Por lo tanto, es lógico pensar que es necesario un esfuerzo (en varios sentidos: en clave de recursos humanos y financieros, pero también -y no es menos importante- en inventiva y valentía) por parte de los socios. Un esfuerzo que debe ser enfocado en el marco de una actuación a medio-largo plazo $\mathrm{y}$, por tanto, que no podía ser de ninguna manera cuantificada ni evaluada, ni mucho menos monitorizada, durante el proyecto EMbleMatiC.

La actuación del CETT en el proyecto no se ha limitado exclusivamente a poner un instrumento práctico a disposición de actores que impulsan el desarrollo turístico sostenible a escala local. Mediante dicho instrumento, lo que se buscaba era poner sobre la mesa una serie de cuestiones que son harto conocidas en el mundo académico, pero que hasta ahora estaban 
poco o nada presentes en la agenda de actores que gestionan los recursos turísticos en ámbitos rurales y de montaña. Son cuestiones relacionadas con la sostenibilidad en múltiples sentidos: el seguimiento de la gestión de establecimientos turísticos mediante indicadores y planes de acción; el fomento de una gastronomía más respetuosa con los recursos locales; la búsqueda de estrategias para paliar los efectos negativos de la movilidad que supone el turismo (transporte público, compensación de la huella de carbono). Éramos (al inicio del proyecto) y seguimos siendo conscientes de la enorme dificultad que supone la puesta en marcha de dichos temas. Pero, al mismo tiempo, estamos seguros que una manera de avanzar es abriendo nuevos ámbitos de actuación y poniendo herramientas a disposición de aquellos en cuyas manos está la posibilidad de avanzar, en este caso desde las montañas mediterráneas, hacia una actividad turística más y mejor integrada en el funcionamiento del territorio a nivel local.

\section{Financiación}

Esta publicación se enmarca dentro del proyecto Interreg "Emblematic Mediterranean Mountains as Coastal Destinations of Excellence", financiado por el programa Interreg MED (referencia: 594 | 1MED15_3.1_M12_272).

\section{Agradecimientos}

Los autores agradecen a la red EMM y a todos los socios del proyecto EMbleMatiC su dedicación, esfuerzo y entusiasmo incondicionales durante todo el desarrollo del mismo. Asimismo, los autores agradecen el trabajo riguroso y el apoyo en el desarrollo del mismo de Eugeni Osácar y Ana Ortega.

\section{Referencias}

Assaf, H.; Baloglu, S. (2016). Motivations and goals of slow tourism. Journal of Travel Research, 55(2), 205-219. http://dx.doi.org/10.1177/0047287514546228

Ávila Bercial, R., y Barrado Timón, D. A. (2005). Nuevas tendencias en el desarrollo de destinos turísticos: marcos conceptuales y operativos para su planificación y gestión. Cuadernos De Turismo, (15), 27-44. Recuperado a partir de https://revistas.um.es/turismo/article/view/18541

Cañizares, M.C. (2013): Sostenibilidad y Turismo: de la documentación internacional a la planificación en España «Horizonte 2020». Boletín de la Asociación de Geógrafos Españoles, 61, 67-92. Recuperado de: https://doi.org/10.21138/bage.1536

Castellanos Verdugo, M. y Orgaz Agüera, F. (2013). Conceptualización y consideraciones en torno al Ecoturismo. Turydes: revista de investigación en turismo y desarrollo local, 6(15). Recuperado de http://www.eumed.net/rev/turydes/15/turismo-sostenible.pdf

Ceballos-Lascurain, H., (1992): Tourism, ecotourism and protected áreas: national parks and protected áreas. IV Congreso sobre Parques Nacionales y Zonas Protegidas, Caracas, Venezuela, 84-89. 
Comisión Europea (2017): El Sistema Europeo de Indicadores Turísticos. Herramienta del ETIS para la gestión de destinos sostenibles. Luxemburgo: Oficina de Publicaciones de la Unión Europea. Recuperado de: https://ec.europa.eu/docsroom/documents/21749.

García Saura, P. J. (2010): Turismo activo y medio ambiente: una implicación necesaria. Aspectos jurídicos. Cuadernos de Turismo, 26, 153-176. Recuperado de: https://revistas.um.es/turismo/article/view/116321

Idelhadj, A.; Rivera, M. y Rodríguez, L- (2012). Turismo responsable, espacios rurales y naturales y cooperación para el desarrollo: a propósito de la" Declaración de Tetuán"(Marruecos). Pasos. Revista de Turismo y Patrimonio Cultural, 10 (5), 651-664.

Lanquar, R. y Rivera, M. (2010). El proyecto “TRES” y la "Declaración de Córdoba” (España): una apuesta por la articulación de estrategias de turismo responsable y solidario desde Europa. Pasos. Revista de Turismo y Patrimonio Cultural, 8 (4), 673-687.

Matos, R. (2004). Can slow tourism bring new life to alpine regions. En K. Weiermair, \& C. Mathies (Eds.): The tourism and leisure industry: Shaping the future. Binghamton, NY: Haworth Hospitality Press, 93-103.

Mulero Mendigorri, A. y Rivera Mateos, M. (2018). Turismo de naturaleza y espacios naturales protegidos en España. Abaco: Revista de cultura y ciencias sociales, 98 (Ejemplar dedicado a: El turismo. Desarrollo, transformación y controversia de un fenómeno social), 84-96

OMT (1999): Guía para las administraciones locales: Desarrollo Turístico Sostenible. Madrid: Organización Mundial del Turismo.

OMT, (2002): El mercado español del ecoturismo. Madrid: Organización Mundial del Turismo.

Pérez, M. (2004): Manual del Turismo Sostenible. Como conseguir un turismo social, económico y ambientalmente responsable. Madrid: Mundi-Prensa.

Sancho Reinoso, A. y Serrat i Mulà, R. (2019a): Experimentation report. Recuperado de: https://emblematic.interreg-

med.eu/fileadmin/user_upload/Sites/Sustainable_Tourism/Projects/EMbleMatiC/D_4.5.1_Ex perimentation_Report.pdf.

Sancho Reinoso, A. y Serrat i Mulà, R. (2019b): Transfer Guide. Recuperado de: https://emblematic.interreg-

med.eu/fileadmin/user_upload/Sites/Sustainable_Tourism/Projects/EMbleMatiC/D532-V3TRANSFER_GUIDE-PP8-CETT-191009.pdf.

Rivera Mateos, M. y Rodríguez García, L. (2012). Turismo responsable, sostenibilidad y desarrollo local comunitario. Córdoba: Universidad de Córdoba. Cátedra Intercultural

Styles, D.; Schönberger, H.; Gálvez Martos, J.L. (2013): Best Environmental Management Practice in the Tourism Sector. Sevilla: Comisión Europea, Joint Research Centre Institute for Prospective Technological Studies, doi:10.2788/33972. Recuperado de: https://susproc.jrc.ec.europa.eu/activities/emas/documents/TourismBEMP.pdf.

Torres Delgado, A. y López Palomeque, F. (2011): The growth and spread of the concept of sustainable tourism: The contribuiton of institutional initiatives to tourism policy. Tourism Management Perspectives, 4, 1-10.

WCED (1987). From One Earth to One World. Our Common Future: Report of the World Commission on Environment and Development. Oxford: Oxford University Press. 